\title{
Politique
}

\section{Index des ouvrages recensés}

Numéro 17, hiver 1990

Les nouveaux enjeux du politique

URI : https://id.erudit.org/iderudit/040664ar

DOI : https://doi.org/10.7202/040664ar

Aller au sommaire du numéro

Éditeur(s)

Société québécoise de science politique

ISSN

0711-608X (imprimé)

1918-6584 (numérique)

Découvrir la revue

Citer ce document

(1990). Index des ouvrages recensés. Politique, (17), 199-212.

https://doi.org/10.7202/040664ar

Ce document est protégé par la loi sur le droit d'auteur. L'utilisation des services d'Érudit (y compris la reproduction) est assujettie à sa politique d'utilisation que vous pouvez consulter en ligne.

https://apropos.erudit.org/fr/usagers/politique-dutilisation/
Cet article est diffusé et préservé par Érudit.

Érudit est un consortium interuniversitaire sans but lucratif composé de l’Université de Montréal, l'Université Laval et l'Université du Québec à Montréal. Il a pour mission la promotion et la valorisation de la recherche. https://www.erudit.org/fr/ 


\title{
Index des ouvrages recensés
}

\author{
$\mathrm{N}^{\circ} 1$ à 16, par ordre alphabétique
}

Devant l'histoire. Les documents de la controverse sur la singularité de l'extermination des Juifs par le régime nazi, Paris, Éditions du Cerf, 1988, par Roger Charland, n 14, 194198.

AMIN, S., ARRIGHI G., FRANK, A.G., WALLERSTEIN, I., La crise, quelle crise?, Paris, Maspero, 1982, par Denis Perreault, $n^{\circ} 6,200-204$.

AMPLEMAN, Gisèle et al., Pratique de conscientisation: expériences d'éducation populaire au Québec, Montréal, Nouvelle Optique, 1983, par Caroline Andrew, $n^{\circ}$ 5, 162-165.

ANASTASSOPOULOS, Jean-Pierre, BLANC, George et DUSSAUGE, Pierre, Les multinationales publiques, Paris, PUF, 1985, par Waldimir Andreff, $n^{\circ}$ 9, 215-221.

ANDREW, Caroline et al., Une communauté en colère: la grève contre Amoco Fabrics à Hawkesbury en 1980, Hull, Éditions Asticou, 1986, par Serge Côté, n 11, 192-198.

ARDAGH, John, Les Allemands, Paris, Pierre Belfond, 1988, par Serge Gosselin, ${ }^{\circ} 14,198-201$.

ARON, Raymond, Le spectateur engagé: entretiens avec JeanLouis Missika et Dominique Wolton, Paris, Julliard, 1981, par Gérard Bergeron, $n^{\circ} 2,183-194$. 
ATTALI, Jacques, Histoire du temps, Paris, Fayard, 1982, par Jean-Yves Chouinard, $n^{\circ}$ 6, 205-209.

AUGER, Geneviève et LAMOTHE Raymonde, De la poêle à frire à la ligne de feu, Montréal, Boréal Express, 1981, par JulesPascal Venne, $n^{\circ}$ 5, 130-133.

BACCIGALUPO, Alain et RHÉAUME, Luc, Les administrations municipales québécoises des origines à nos jours - Tome 1: Les municipalités, Montréal, Agence d'Arc, 1984, par Maurice Couture, $\mathrm{n}^{\circ}$ 8, 165-168.

BALTHAZAR, Louis, Bilan du nationalisme au Québec, Montréal, L'Hexagone, 1986, par Gérald Bernier, n 11, 171-176.

BEAUD, Michel et LATOUCHE, Daniel, L'art de la thèse, Montréal, Boréal, 1988, par Denis Monière, $\mathrm{n}^{\circ}$ 14, 201-204.

BÉGIN, Monique, L'assurance-santé: plaidoyer pour le modèle canadien, Montréal, Boréal Express, 1987, par Michel Pelletier, $\mathrm{n}^{\circ} 14,171-178$.

BÉLANGER, André-J., Framework for a Political Sociology, Toronto, University of Toronto Press, 1985, par Micheline de Sève, $n^{\circ} 10,133-137$.

BELLANGER, Réal, L'impossible défi-Albert Sévigny et les conservateurs fédéraux (1902-1918), Québec, Presses de 1'Université Laval, 1983, par Michel Martin, n 6, 181-186.

BÉLANGER, Yves et FOURNIER, Pierre, L'entreprise québécoise - Développement historique et dynamique contemporaine, Québec, Hurtubise HMH, 1987, par L. Jacques Filion, $n^{\circ} 14,163-167$.

BÉLANGER, Yves, BRUNELLE, Dorval (Eds), L'Ėre des libéraux. Le pouvoir fédéral de 1963 à 1984, Sillery, Presses de l'Université du Québec, 1988, par Chantal Maillé, n 16, 148154.

BELLAVANCE, Michel, Les politiques gouvernementales: élaboration-gestion-évaluation, Montréal, Agence d'Arc, 1985, par Laurent Lepage, $n^{\circ}$ 9, 203-205.

BELlEMARE, Diane et SAINT-PIERRE, Céline (Eds), Les stratégies de reprise, Montréal, Éditions Albert Saint-Martin, 1984, par Raymond Hudon, $n^{\circ}$ 9, 209-215. 
BERGERON, Claude et al., Les choix géopolitiques du Canada. L'enjeu de la neutralité, Montréal, Éditions du Méridien, 1988, par Bruno Munier, $\mathrm{n}^{\circ} 15,146-149$.

BERGERON, Gérard, À nous autres - aide-mémoire politique par le temps qui court, Montréal, Québec/Amérique, 1986, par François Rocher, $\mathrm{n}^{\circ} 11,167-171$.

BERGERON, Gérard, La guerre froide recommencée, Montréal, Boréal Express,1986, par Gérard Chaliand, ${ }^{\circ} 11,190-192$.

BERGERON, Gérard, Notre miroir à deux faces, Montréal, Québec/ Amérique, 1985, par Louis Massicotte, $\mathrm{n}^{\circ}$ 8, 171-174.

BERGERON, Gérard, Pratique de l'État au Québec, Montréal, Québec/Amérique, 1984, par Jean-Pierre Gaboury, nº 6, 173178.

BERNARD, Louis, Réflexions sur l'art de se gouverner, Montréal, ENAP/Québec-Amérique, 1987, par Pier Bouchard, $n^{\circ} 12$, 129-132.

BERNIER, Gérald et BOILY, Robert, Le Québec en transition: 1760-1867, Bibliographie thématique, Montréal, ACFAS, 1987, par Serge Denis, $n^{\circ} 13,152-156$.

BERNIER, Gérald et BOISMENU, Gérard (Eds), Crise économique, transformations politiques et changements idéologiques, Montréal, Cahiers de l'ACFAS, $\mathrm{n}^{\circ} 16,1983$, par Paul Pilisi, $n^{\circ} 6,195-200$.

BERNIER, Gérald, BOILY, Robert, avec la participation de SALEE, Daniel, Le Québec en chiffres, de 1850 à nos jours, Montréal, ACFAS, 1986, par Serge Denis, $n^{\circ} 13,152-156$.

BERNIER, Robert, Le marketing gouvernemental au Québec: 1929-1985, Montréal, Gaëtan Morin éditeur, 1988, par Daniel Latouche, $\mathrm{n}^{\circ} 16,125-130$.

BLOUIN, Jean, BERGERON, Gérard, De l'autre côté de l'action, Montréal, Nouvelle Optique, 1982, par Jacques Gagnon, $\mathrm{n}^{\circ} 3,157-160$.

BOISMENU, Gérard (Ed.), Espace régional et nation, Montréal, Boréal Express, 1983, par François Houle, $n^{\circ} 4,171-175$.

BOISMENU, Gérard, Le duplessisme, Montréal, Presses de l'Université de Montréal, 1981, par Lizette Jalbert, $\mathrm{n}^{\circ} 1,102$ 109. 
BOIVIN, Dominique, Le lobbying ou le pouvoir des groupes de pression, Montréal, Éditions du Méridien, 1984, par Jean Dion, $\mathrm{n}^{\circ} 7,123-127$.

BONIN, Bernard, L'entreprise multinationale et l'État: un exemple du couple fascination-répulsion, Montréal, Éditions Études vivantes, 1984, par Johane Bergeron, $n^{\circ} 7,133-143$.

BOUCHARDEAU, Huguette, Un coin dans leur monde, Paris, Syros, 1980, par Anne-Marie Gingras, $n^{\circ}$ 5, 146-148.

BOUDREAU, Emest, Le rêve inachevé. Le PQ, l'indépendance et la crise, Montréal, Nouvelle Optique, 1983, par François Houle, $n^{\circ} 6,186-187$.

BOURASSA, Guy et LEVEILLÉE, Jacques (Eds), Le système politique de Montréal, Montréal, ACFAS, 1986, par Caroline Andrew, $\mathrm{n}^{\circ} 11,202-205$.

BOURBEAU, Robert et LÉGARÉ, Jacques, Évolution de la mortalité au Canada et au Québec, 1831-1931. Essai de mesure par génération, Montréal, Presses de l'Université de Montréal, 1982, par Rénald Gravel, $n^{\circ} 4,187-188$.

BOURQUE, Gilles et DUCHASTEL, Jules, Restons traditionnels et progressifs. Pour une nouvelle analyse du discours politique. Le cas du régime Duplessis au Québec, Montréal, Boréal, 1988, par Réjean Pelletier, $\mathrm{n}^{\circ}$ 15, 111-113.

BRUNELLE, Dorval, Les trois colombes, Montréal, VLB, 1985, par Yves Bélanger, $\mathrm{n}^{\circ}$ 9, 205-209.

BRUNELLE, Dorval, Socialisme, étatisme et démocratie, Montréal, Éditions Albert Saint-Martin, 1983, par Gilles Labelle, $\mathrm{n}^{\circ} 9,176-181$.

CAMBROSIO, A. et DUCHESNE, R. (Eds), Science, technologie et société. Les enjeux du progrès, Sainte-Foy, Presses de l'Université du Québec et Télé-Université, 1984, par Réjean Landry, $n^{\circ} 8,144-146$.

CAMERON, Duncan et HOULE, François (Eds), Le Canada et la nouvelle division internationale du travail, Ottawa, Éditions de l'Université d'Ottawa, 1985, par Christian Deblock, $n^{\circ} 8,146-153$. 
CHANT, C. et HOGG, I., La bombe: armes et scénarios militaires, Paris, Autrement, 1983, par Michel Fortmann, ${ }^{\circ} 7,148$ 153.

COHEN, Yolande (Eds), Femmes et contre-pouvoirs, Montréal, Boréal Express, 1987, par Linda Cardinal, n 13, 160-165.

COLLECTIF, Terre des femmes, Paris, Montréal, Maspéro/Boréal Express, 1983, par Nadine Jammal, $n^{\circ}$ 5, 149-151.

COMMISSION DE LA REPRÉSENTATION ÉLECTORALE, Pour un mode de scrutin équitable. La proportionnelle territoriale, Québec, Gouvernement du Québec, 1984, par Robert Boily, $n^{\circ} 6,167-173$.

CORTEN, André et TAHON, Marie-Blanche, La radicalité du quotidien. Communauté et informatique, Montréal, VLB Éditeur, 1987, par Jean-Jacques Gislain, n 13, 166-169.

CRÊTE, Jean (Eds), Comportement électoral au Québec, Chicoutimi, Gaëtan Morin éditeur, 1984, par Conrad Winn, $n^{\circ} 9$, 201-203.

DE CLOSETS, François, Toujours plus, Paris, Grasset, 1982, par Denis Monière, $\mathrm{n}^{\circ} 3,145-149$.

DE SÈVE, Micheline, Pour un féminisme libertaire, Montréal, Boréal Express, 1985, par Hélène Sarrasin, n 9, 193-197.

DEBOUZY, Marianne, Travail et travailleurs aux États-Unis, Paris, Éditions La Découverte, 1984, par Serge Denis, n 7, 159. 164.

DEBRAY, Régis, Critique de la raison politique, Paris, Gallimard, 1981, par Yoland Sénécal, n 3, 152-156.

DELAPIERRE, Michel et ZIMMERMAN, Jean-Benoît, L'informatique du Nord au Sud, Paris, La documentation française, 1986, par Gérard Boismenu, n 11, 205-208.

DELORME, Pierre, L'ordre urbain: administration publique de l'espace urbain au Québec, Hull, Éditions Asticou, 1986, par Pierre Filion, $\mathrm{n}^{\circ} 12,140-145$.

DENIS, Serge, Un syndicalisme pur et simple: mouvements ouvriers et pouvoir politique aux États-Unis, 1919-1939, Montréal, Boréal Express, 1986, par Gregor Murray, $n^{\circ} 11$, 211-215. 
DESLAURIERS, Jean-Pierre (Eds), Les méthodes de la recherche qualitative, Sillery, Presses de l'Université du Québec, 1987, par Lawrence Olivier, ${ }^{\circ} 12,159-162$.

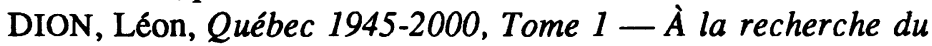
Québec, Québec, PUL, 1987, par Guy Rocher, n 14, 159163.

DION, Stéphane, La politisation des mairies, Paris, Économica, 1986, par Jacques Léveillée, $\mathrm{n}^{\circ} 12,138-140$.

DOBRY, Michel, Sociologie des crises politiques, Paris, Presses de la Fondation nationale des sciences politiques, 1986, par Marcel Fournier, $\mathrm{n}^{\circ} 12,123-129$.

DONNEUR, André, L'Alliance fragile. Socialistes et communistes français, 1922-1983, Montréal, Nouvelle Optique, 1984, par George Ross, $\mathrm{n}^{\circ}$ 8, 177-179.

DOSTALER, Gilles et ÉTHIER, Diane (Eds) Friedrich Hayek. Philosophie, économie et politique, Paris, Économica, 1988, par France Giroux, $\mathrm{n}^{\circ} 16,134-140$.

DUHAMEL, Luc, Les Soviétiques et les voies de la révolution en Europe occidentale (de Lénine à Brejnev), Montréal et Paris, Presses de l'Université de Montréal et Éditions Antrhopos, 1981, par Miron Rezun, n 6, 191-195.

DUMONT, René, Finis les lendemains qui chantent... La Chine décollectivise, Paris, Seuil, 1984, par Rodolphe de Koninck, $\mathrm{n}^{\circ} 7,143-148$.

DUPUIS, Monique, Crise mondiale et aide internationale - Stratégie canadienne et développement du Tiers-monde, Montréal, Nouvelle Optique, 1984, par Sylvain Ayotte, $n^{\circ} 7$, 128-133.

EN COLLABORATION, L'économie fiction: contre les nouveaux économistes, Paris, Maspéro, 1982, par Benoît Godin, $\mathrm{n}^{\circ} 3,149-152$.

EUDES, Yves, La conquête des esprits, Paris, Maspéro, 1982, par Denis Monière, $\mathrm{n}^{\circ}$ 2, 176-179.

FAUCHER, Philippe et BERGERON, Johanne, Hydro-Québec, la société de l'heure de pointe, Montréal, Presses de l'Université de Montréal, 1986, par Jeanne Kirk Laux, $n^{\circ} 11$, 163-166. 
FAYE, Jean-Pierre, Dictionnaire politique portatif en cinq mots, Paris, Gallimard, coll. Idées, 1982, par Denis Monière, $\mathrm{n}^{\circ} 4,176-178$.

FILION, Gérard, Fais ce que peux, Montréal, Boréal, 1989, par Gérard Bergeron, $\mathrm{n}^{\circ}$ 14, 107-111.

FRASER, Graham, Le Parti québécois, Montréal, Libre Expression, 1984, par Jean-Pierre Beaud, $n^{\circ} 8,160-162$.

GAGNON, Alain G. (Ed.), Les opérations-dignité: naissance d'un mouvement social dans l'est du Québec, Montréal, Leméac/ GRIDEQ/Carleton University, 1981, par Jean-Louis Gendron, $n^{\circ} 2,179-181$.

GAGNON, Alain G., Développement régional, État et groupes populaires, Hull, Éditions Asticou, 1985, par Denis Perreault, $\mathrm{n}^{\circ} 8,163-165$.

GALBRAITH, John Kenneth, Anatomie du pouvoir, Paris, Seuil, 1985, par Vincent Lemieux, $\mathrm{n}^{\circ} 8,174-176$.

GAY, Daniel, Les élites québécoises et l'Amérique latine, Montréal, Nouvelle Optique, 1983, par Yvon Grenier et Jean Daudelin, $n^{\circ} 6,188-191$.

GODBOUT, Jacques, La participation contre la démocratie, Montréal, Albert Saint-Martin, 1983, par André Vachet, $\mathrm{n}^{\circ} 7,165-170$.

GOSSELIN, Guy (Eds), La politique étrangère du Canada: approches bilatérale et régionale, Québec, CQRI, 1984, par JeanFrançois Rioux, $\mathrm{n}^{\circ}$ 8, 153-155.

GOULDNER, Alvin W., The Future of Intellectuals and the Rise of the New Class, New York, The Seabury Press, 1979, par Benoît Allaire, $\mathrm{n}^{\circ} 1,109-114$.

GOUVERNEMENT DU QUÉBEC, Le Québec dans le monde. État de la situation. Les conférences socio-économiques du Québec, Québec, Gouvernement du Québec, 1984, par Guy Gosselin, $n^{\circ} 7,114-118$.

GOW, James Iain et al., Introduction à l'administration publique: une approche politique, Chicoutimi, Gaëtan Morin éditeur, 1987, par Antoine Ambroise, $n^{\circ} 13,146-150$.

GOW, James Iain, Histoire de l'administration publique québécoise, 1867-1970, Montréal, Toronto, Presses de l'Univer- 
sité de Montréal/Institut d'administration publique du Canada, 1986, par Marcel Proulx, $n^{\circ} 13,139-142$.

GUILLEMIN, Henri, Le Général clair-obscur, Paris, Seuil 1984, par Yoland Sénécal, $n^{\circ}$ 7, 170-176.

HAMEL, Pierre, LEONARD, Jean-François et MAYER, Robert, Les mobilisations populaires urbaines, Montréal, Nouvelle Optique, 1982, par Caroline Andrew, n 5, 162-165.

HAMMER, Richard, Vatican Connection, Paris, Éditions Balland, 1982, par Jean-Paul Montminy, ${ }^{\circ} 4$, 184-187.

HAMON, Hervé et ROTMAN, Patrick, Les intellocrates - Expédition en haute intelligentsia, Paris, Éditions Ramsay, 1981, par Michèle Lamont, $n^{\circ} 1,114-117$.

HAROUEL, Jean-Louis, Essai sur l'inégalité, Paris, PUF, 1984, par Douglas Moggach, $\mathrm{n}^{\circ}$ 9, 185-189.

HERO, Alfred O. et DANEAU, Marcel (Eds), Problems and Opportunities in US - Quebec Relations, Boulder, Colorado, Westview Press, 1984, par François-Pierre Gingras, $n^{\circ} 7$, 120-122.

HERVOUET, G. et GAlARNEAU, H. (Eds), Présence internationale du Québec: chronique des années 1978-1983, Québec, CQRI, 1984, par P. Soldatos, $\mathrm{n}^{\circ}$ 7, 119-120.

JALBERT, Lizette et BEAUDRY, Lucille (Eds), Les métamorphoses de la pensée libérale: sur le néo-libéralisme actuel, Montréal, Presses de l'Université du Québec, 1987, par Max Nemni, $n^{\circ} 14,179-184$.

JALBERT, Lizette et LEPAGE, Laurent (Eds), Néo-conservatisme et restructuration de l'État, Montréal, Presses de l'Université du Québec, 1986, par J. Yvon Thériault, $n^{\circ} 11,159-163$.

JOURNEES «ELLES VOIENT ROUGE», Féminisme et marxisme, Paris, Éditions Tierce, 1980, par Hélène Massé, $n^{\circ}$ 5, 151157.

JUDT, Tony, Le marxisme et la gauche française, 1830-1981, Paris, Hachette, 1987, par Joceline Chabot, $\mathrm{n}^{\circ} 14,191-$ 194.

KIRK LAUX, Jeanne et MOLOT, Maureen, State Capitalism - Public Enterprise in Canada, London et Ithaca: Cornell University Press, 1988, par Yves Bélanger, n 14, 167-171. 
KORANY, Bahgat et collaborateurs, Analyse des relations internationales - approches, concepts et données, Montréal/Québec, Gaëtan Morin/Centre québécois de relations internationales, 1987, par Pierre Martin, $n^{\circ} 15,125-127$.

LA ROCHELLE, Louis, En flagrant délit de pouvoir. Chronique des événements politiques de Maurice Duplessis à René Lévesque, Montréal, Boréal Express, 1982, par René Gravel.

LACROIX, Philippe (Eds) Éviter la guerre? Réponses à quelques questions sur les risques de guerre, Paris, Maspéro, 1983, par Michel Fortmann, $\mathrm{n}^{\circ}$ 7, 148-153.

LAFRANCE, Jean-Paul, La télévision: un média en crise, Montréal, Québec/Amérique, 1982, par René Vézina, n² 2, 194198.

LAMOUREUX, André, Le NPD et le Québec, 1958-1985, Montréal, Éditions du Parc, 1985, par Serge Denis, n 10,129 133.

LAMOUREUX, Diane, Fragments et collage. Essai sur le féminisme québécois des années 70, Montréal, Remue-Ménage, 1986, par Denyse Côté, $\mathrm{n}^{\circ} 11,182-185$.

LAPLANTE, Pierre et LEVY, Joseph (Eds), La paix: nouvelles avenues, Montréal, Éditions du Méridien, 1987, par France Giroux, $\mathrm{n}^{\circ} 14,187-190$.

LAURENT, Alain, De l'individualisme - Enquête sur le retour de l'individu, Paris, PUF, 1985, par Claire Frémont, $\mathrm{n}^{\circ} 9$, 181-185.

LE COLLECTIF CLIO, L'histoire des femmes au Québec depuis quatre siècles, Montréal, Quinze, 1982, par Lise Brassard, $n^{\circ}$ 5, 125-129.

LE NET, Michel, L'État annonceur: techniques, doctrine et morale de la communication sociale, Paris, Les Éditions d'organisation, 1981, par Michelle Massie, $n^{\circ} 2,198-200$.

LECLERC, Michel, La science politique au Québec, Montréal, L'Hexagone, 1982, par André-J. Bélanger, n 4, 188-196.

LEFORT, Claude, Essais sur le politique, XIXe-XXe siècles, Paris, Seuil, 1986, par Pierre Boyer, $\mathrm{n}^{\circ} 11,185-189$. 
LEMIEUX, Vincent, Les sondages et la démocratie, Québec, Institut québécois de recherche sur la culture, 1988, par JeanPierre Beaud, $\mathrm{n}^{\circ} 15,122-124$.

LEMIEUX, Vincent, Systèmes partisans et partis politiques, Montréal, PUQ, 1985, par Luc Fortin, n 9, 197-200.

LEROUX, Georges et VAN SCHENDEL, Michel (Eds), Sédiments, Montréal, Hurtubise HMH, 1986, par Anne Legaré, $n^{\circ} 11$, 208-211.

LÉVESQUE, Andrée, Virage à gauche interdit - Les communistes, les socialistes et leurs ennemis au Québec, 1929-1939, Montréal, Boréal Express, 1984, par Nicole Lemire, $n^{\circ} 8$, 168-171.

LÉVESQUE, René, Attendez que je me rappelle..., Montréal, Québec/Amérique, 1986, par Gérard Bergeron, n 11, 176182.

LIEBICH, André, Le libéralisme classique, Montréal, PUQ, 1985, par J. Yvon Thériault, $\mathrm{n}^{\circ}$ 9, 189-193.

LINTEAU, P.A. et al., Histoire du Québec contemporain: le Québec depuis 1930, Montréal, Boréal Express, 1986, par Daniel Salée, $\mathrm{n}^{\circ} 12,148-154$.

LIPIETZ, Alain, L'audace ou l'enlisement, Paris, Éditions la Découverte, 1985, par Diane Éthier, $n^{\circ} 10,145-148$.

LIPIETZ, Alain, Mirages et miracles: problèmes de l'industrialisation dans le Tiers-monde, Paris, Édition la Découverte, 1985, par Diane Éthier, $\mathrm{n}^{\circ} 10,141-145$.

LOWE, Graham S., Women in the Administrative Revolution, Toronto, University of Toronto Press, 1987, par Irène Lépine, $\mathrm{n}^{\circ} 13,142-146$.

MACPHERSON, C.D., Principes et limites de la démocratie libérale, Montréal/Paris, Boréal Express/la Découverte, 1985, par André Vachet, $n^{\circ}$ 9, 169-176.

MARCIL-LACOSTE, Louise, La raison en procès, Montréal, Hurtubise HMH, 1987, par Paul Saint-Hilaire, $\mathrm{n}^{\circ} 16,140-144$. MARCOU, Lilly, Les défis de Gorbatchev, Paris, Plon, 1988, par Michel Roche, $\mathrm{n}^{\circ} 15,128-132$. 
MASSICOTTE, Louis et BERNARD, André, Le scrutin au Québec: un miroir déformant, Montréal, HMH, 1985, par André Blais, $\mathrm{n}^{\circ} 10,119-122$.

McROBERTS, Kenneth et POSTGATE, Dale, Développement et modernisation du Québec, Montréal, Boréal Express, 1982, par Réjean Pelletier, $n^{\circ} 4,167-169$.

MERCIER, Jean, Les Québécois entre l'État et l'entreprise, Montréal, L'Hexagone, 1988, par Pierre P. Tremblay, $\mathrm{n}^{\circ} 15$, 143-146.

MONIÈRE, Denis et GUAY, Jean-H., Introduction aux théories politiques, Montréal, Québec/Amérique, 1987, par Réjean Landry, $\mathrm{n}^{\circ} 13,150-152$.

MOREAU, François, Le développement international des banques canadiennes, Montréal, Albert Saint-Martin, 1985, par Bernard Élie, $n^{\circ} 10,137-141$.

MORIN, Claude, L'art de l'impossible: la diplomatie québécoise depuis 1960, Montréal, Boréal Express, 1987, par Hélène Galarneau, $\mathrm{n}^{\circ} 12,132-138$.

MORIN, Claude, Les lendemains piégés - Du référendum à la nuit des longs couteaux, Montréal, Boréal Express, 1987, par Hélène Galarneau, $n^{\circ} 12,132-138$.

MORIN, Richard, Réanimation urbaine et pouvoir local, Montréal, Presses de l'Université du Québec/INRS - Urbanisation, 1987, par Louise Quesnel, ${ }^{\circ} 12,145-148$.

MOSSUZ-LAVAU, J. et SINEAU, M., Enquête sur les femmes et la politique en France, Paris, PUF, 1983, par Marie-Blanche Tahon, $n^{\circ} 5,140-146$.

MOSSUZ-LAVAU, J. et SINEAU, M., Les femmes françaises en 1978, insertion sociale, insertion politique, Paris, Rapport pour le CORDES, mars 1980, par Marie-Blanche Tahon, $n^{\circ} 5,140-146$.

NEMO, Philippe, La société de droit selon F.A. Hayek, Paris, PUF, 1988, par France Giroux, $\mathrm{n}^{\circ}$ 16, 134-140.

OLIVIER, Christiane, Les enfants de Jocaste: l'empreinte de la mère, Paris, Denoël/Gonthier, 1980, par Nathaly Gagnon, $n^{\circ} 5,157-162$. 
ORBAN, Edmond et coll., Le système politique des États-Unis, Montréal, Presses de l'Université de Montréal, 1987, par Daniel Latouche, $\mathrm{n}^{\circ} 13,158-160$.

ORBAN, Edmond, La dynamique de la centralisation dans l'État fédéral: un processus irréversible?, Montréal, Québec/ Amérique, 1984, par André Bernard, n 10, 122-124.

PELLETIER, Réjean, Partis politiques et société québécoise - De Duplessis à Bourassa, 1944-1970, Montréal, Québec/Amérique, 1989, par Guy Lachapelle, $\mathrm{n}^{\circ} 16,130-133$.

PIOTTE, Jean-Marc, La communauté perdue. Petite histoire des militantismes, Montréal, VLB Éditeur, 1987, par Pierrette Bouchard, $\mathrm{n}^{\circ} 13,156-158$.

PLASSE, Micheline, Santé et sécurité au travail: de la confrontation à la concertation, Montréal, Agence d'Arc, 1988, par Yves Martin, $\mathrm{n}^{\circ} 15,119-122$.

PORTELLI, Hugues (Eds), L'internationale socialiste, Paris, Éditions ouvrières, 1983, par André Beaudet, $n^{\circ} 7,153-$ 159.

RATZEL, Friedrich, La géographie politique: les concepts fondamentaux, Paris, Fayard, 1987, par Jean-François Rioux, $n^{\circ} 13,177-180$.

RÉMOND, René, Les droites en France, Paris, Éditions AubierMontaigne, 1982, par Yoland Sénécal, n 4, 178-184.

ROBITAILLE, Louis-Bernard, Erreurs de parcours, Montréal, Boréal Express, 1982, par Jacques Gagnon, $\mathrm{n}^{\circ} 2,182-183$.

ROSANVALLON, Pierre, La crise de l'État-providence, Paris, Seuil, 1981, par Gilles Breton, n 3, 160-163.

ROUILlARD, Jacques, Histoire du syndicalisme au Québec. Des origines à nos jours, Montréal, Boréal, 1989, par Roch Denis, $\mathrm{n}^{\circ} 16,144-148$.

RUFFAT, Michèle, Le contre-pouvoir consommateur aux ÉtatsUnis, Paris, PUF, 1987, par François Rocher, $n^{\circ} 13,172$ 176.

SARGENT, Lydia (Ed.), Women and Revolution. A Discussion of the Unhappy Marriage of Marxism and Feminism, Montréal, Black Rose Books, 1981, par Hélène Massé, $n^{\circ} 5$, 151-157. 
SAVARY, Claude (Ed.), Les rapports culturels entre le Québec et les États-Unis, Québec, Institut québécois de recherche sur la culture, 1984, par Daniel Bonin, $n^{\circ} 10,125-129$.

SAVOIE, Donald J., Regional Economic Development: Canada's Search for Solutions, Toronto, Presses de l'Université de Toronto, 1986, par Alain G. Gagnon, n 11, 198-202.

SIMARD, Carolle, L'administration contre les femmes, Montréal, Boréal Express, 1983, par James Iain Gow, $n^{\circ}$ 5, 136-140.

SINEAU, Mariette, Des femmes en politique, Paris, Economica, 1988, par Caroline Andrew, $\mathrm{n}^{\circ}$ 14, 184-186.

SOULET, Marc Henry, Le silence des intellectuels, Montréal, Éditions Saint-Martin, 1987, par Stephen Brooks, $n^{\circ} 13$, 169-171.

SYMONS, Gladys L. (Eds), La culture des organisations, Québec, Institut québécois de recherche sur la culture, 1988, par Marcel Belleau, $\mathrm{n}^{\circ} 15,132-136$.

THÉRIAULT, J. Yvon, La société civile ou la chimère insaisissable, Montréal, Québec/Amérique, 1985, par Jean-Guy Vaillancourt, $\mathrm{n}^{\circ} 15,140-143$.

TINKISS, GOOD Mabel, Lionel Chevrier, Montréal, Stanké, 1987, par Michel Sarra-Bournet, $n^{\circ} 12,154-159$.

TOURAINE, Alain, Le retour de l'acteur, Paris, Fayard, 1984, par Carol Levasseur, $n^{\circ} 8,134-143$.

VANDELAC, Louise et al, Du travail et de l'amour. Les dessous de la production domestique, Montréal, Albert Saint-Martin, 1985, par Denyse Côté, ${ }^{\circ}$ 8, 155-159.

VINCENTHIER, Georges, Histoire des idées au Québec, 18371980, Montréal, VLB éditeur, 1983, par Jacques Gagnon, $\mathrm{n}^{\circ} 6,178-180$.

VITALIS, André, Informatique, pouvoir et libertés, Paris, Economica, 1981, par Benoît Gauthier, n² 2, 169-176.

WHITE, Julie, Les femmes et le travail à temps partiel, Ottawa, Conseil consultatif canadien de la situation de la femme, 1983, par Céline Saint-Pierre, $n^{\circ}$ 5, 133-136.

ZYLBERBERG, Jacques (Eds), Masses et postmodernité, Québec, Presses de l'Université Laval, 1986, par Nassib El Hussei$\mathrm{ni}, \mathrm{n}^{\circ} 15,136-140$. 


\section{Synthèses bibliographiques/Notes critiques}

BERGERON, Johanne, L'autel du libéralisme: une revue des rapports Fortier, Gobeil et Scowen, $\mathrm{n}^{\circ} 11,129-138$.

BERGERON, Johanne, L'informatisation de la société: une réflexion critique autour de quelques ouvrages récents, $\mathrm{n}^{\circ} 2$, 153-165.

BRETON, Gilles, Un regard critique sur l'économie mondiale, $\mathrm{n}^{\circ} 7$, 101-114.

СНАВОТ, Marc, Sur le développement international des banques canadiennes, $\mathrm{n}^{\circ} 11,147-156$.

FILION, Jacques, Les livres sur Trudeau, $\mathrm{n}^{\circ}$ 1, 95-102.

HUDON, Raymond, Au-delà de la crise budgétaire péquiste, $\mathrm{n}^{\circ} 3$, 131-141.

MOCKLE, Daniel, La rationalité juridique et politique de l'Étatprovidence, $\mathrm{n}^{\circ} 11,138-146$.

PARADEISE, Catherine, Fortune et infortune de la femme mariée de François de Sigly, ${ }^{\circ} 16,111-121$. 